\title{
Research on Strategic Supplier Selection and Evaluation Method of Manufacturing Enterprise Based on AHP-GCA Method
}

\author{
Banglei $\mathrm{Wu}^{1, a}$, Xiaojuan Chen ${ }^{1, \mathrm{~b}}$, Jing $\mathrm{Yu}^{1, \mathrm{c}}$ and Qing Sun ${ }^{1, \mathrm{~d}}$ \\ ${ }^{1}$ Economic and Management Department, Anhui Technical College of Mechanical and Electrical \\ Engineering, Wuhu Anhui 241000, China \\ awubanglei@126.com, bcxjahjd@126.com, cyujing625@126.com, 'ahjdsq617@126.com
}

Keywords: strategic supplier, selection and evaluation system, analytical hierarchy process and grey correlation analysis (AHP-GCA)

\begin{abstract}
With the application of intelligence technology in manufacturing industry, the competition of supply chain in manufacturing industry is becoming more and more competitive. Strategic supplier is a key element for the success of manufacturing enterprise. At present, there are many methods for the selection and evaluation of strategic supplier of manufacturing enterprise. Analytical hierarchy process and grey correlation analysis (AHP-GCA) are methods used to choose strategic supplier of manufacturing enterprise by building optimum reference data and solving grey correlation degree. These have positive effects to the enterprise practice in qualitative diagnosis, quantitative diagnosis and grey information processing.
\end{abstract}

\section{Introduction}

The standards of strategic supplier selection and evaluation system have obvious characteristic of hierarchy. The first level standards can be disassembled into different sub-indexes which form tree structure that offers structure basis for analytic hierarchy method. Analytic hierarchy method is a relatively mature theory which has much hands-on experience to draw on. Grey correlation analysis is a method which carries out quantitative description and comparison towards system development and change. Its basic idea is to judge whether the relation between reference sequence of number and comparison sequence of number is close or not by confirming the degree of closeness/ proximity of these two curves, and use grey correlation degree to reflect the proximity between curves and find out the difference and closeness. AHP-GCA (Analytic hierarchy processing and grey correlation analysis) model combine analytic hierarchy processing method with grey correlation analysis method organically. In AHP-GCA (Analytic hierarchy processing and grey correlation analysis) model, different indexes' weight are confirmed by using analytic hierarchy processing method. Human's subjective judgment is expressed in number, and consistency check is carried out, thus the side-effect of subjective factors could be brought down, and drastically. Grey correlation analysis method implements whitening processing on qualitative index items (grey indexes) from indexes, and standardizes quantitative index data. By this mean, the losing and deviation of information would be minimized and the correctness of conclusion could be guaranteed.

\section{Selecting Evaluation Model Based on AHP-GCA}

First step, building tree structure model.

Second step, constructing "multiple judgment" matrix, $B_{k}$ signifies number $\mathrm{k}$ index in the B gradation. Suppose k's indexes at this gradation is $B_{1}, \ldots, B_{n}$, its direct upper level index is $A$, then make multiple comparison to $B_{1}, \ldots, B_{n}$ aiming at index $A$ by using nine points marking method. Thus comparison value $b_{i j}$ is appeared. We note down judgment matrix $B=\left(b_{i j}\right)_{n \times n}$. So B's maximum eigenvalue is $\lambda_{\max }$, normalized eigenvector that belongs to $\lambda_{\max }$ is $\omega=\left(\omega_{1}, \cdots, \omega_{n}\right)^{T}$. In this way, $\left(\omega_{1}, \cdots, \omega_{n}\right)$ is weighting of index $B_{1}, \ldots, B_{n}$ to index A. In actual computation, we can calculate 
approximately index relative weighting under every single index by using "extraction of root" method. And after normalization, it is relatively important weighting of indexes at the same gradation to indexes at the direct upper gradation. In the similar way, we calculate from upper gradation to lower gradation until it comes to the last gradation which has its weighting to the direct upper gradation. If we note $D_{k}$ as matrix organized by weighting in column of all indexes in number $\mathrm{k}$ gradation to all indexes in the direct upper gradation, then the combined weighting vector in gradation is:

$$
W^{k}=D_{k} \cdot D_{k-1} \cdots \cdot D_{2} \cdot D_{1}
$$

While getting judgment matrix B, index consistency C.I $_{\text {I }}$ is needed check due to probable existence of subjective judgment inconsistency of nine points marking method.

In it,

$$
\begin{aligned}
& \overline{\omega_{i}}=\sqrt[n]{\prod_{j=1}^{n} b_{i j}} . \\
& \omega_{i}=\frac{\overline{\omega_{i}}}{\sum_{i=1}^{n} \overline{\omega_{i}}} . \\
& C_{.} I=\frac{\lambda_{\max }-n}{n-1} . \\
& \lambda_{\max }=\sum_{i=1}^{n} \frac{\sum_{j=1}^{n} b_{i j} \omega_{j}}{n \omega_{i}} .
\end{aligned}
$$

If $C . I \leq 0.1$, then we judge matrix B is satisfactory[1].

The third step, collecting various evaluation indexes data of back up strategic suppliers, and carrying out standardization on quantitative indexes data, quantitative processing of whitening weight function on qualitative indexes data. After this, the indexes data sequences constitute comparison data sequence of evaluation index of various back up suppliers. Choose the maximum values (optimum values)from various indexes that have been processed to form the optimal reference $\operatorname{data}\left\{Z_{0}\right\}=\left\{z_{o 1}, z_{02}, \cdots z_{0 n}\right\}$.

(1)Standardization of quantitative index data

Extremely large index --the larger the indexes value the better

Extremely small index--the smaller the indexes value the better

Suppose there are $g$ back up suppliers, f indexes. $d_{i j}$ is original data, $y_{i j}$ is $\operatorname{standard~data~}(i=1,2, \cdots$, $\mathrm{g} ; \mathrm{j}=1,2, \cdots, \mathrm{f}) . G_{j}$ and $g_{j}$ are respectively the largest value and the smallest value of number $\mathrm{j}$ index of back up supplier. The indexes data is changed refers with Fig. 1 and Fig. 2 . 


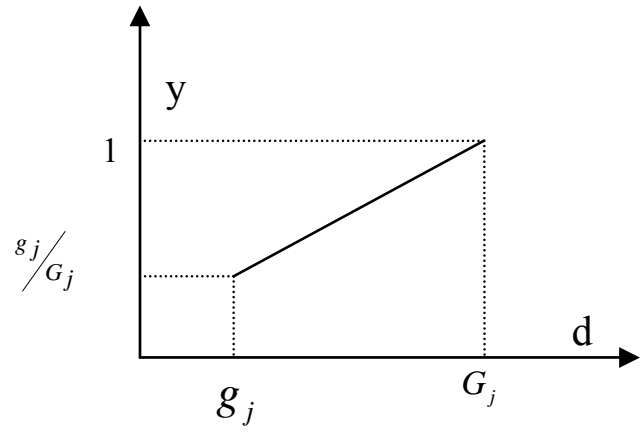

Fig.1 Extremely large type

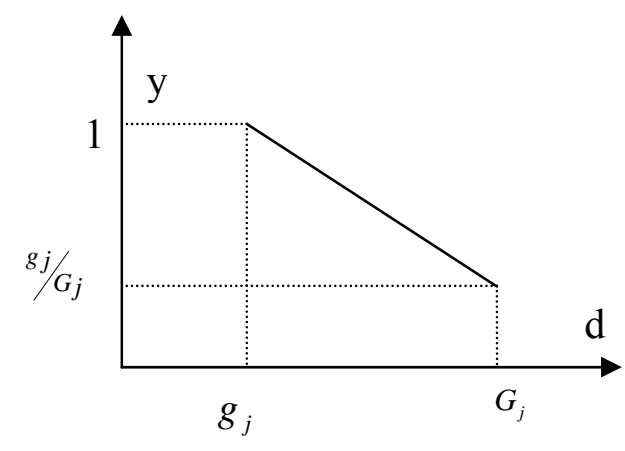

Fig.2 Extremely small type

Extremely large index data conversion:

$y_{i j}=d_{i j} / G_{j} \quad(\mathrm{i}=1,2,3, \ldots \mathrm{g} ; \mathrm{j} \in$ extremely large type $)$.

Extremely small index data conversion:

$y_{i j}=1+g_{j} / G_{j}-y_{i j} / G_{j} \quad(\mathrm{i}=1,2,3, \ldots \mathrm{g} ; \mathrm{j} \in$ extremely small type $)$.

(2) Quantitative processing of whitening weight function on qualitative indexes data

Experts make scores at one qualitative index respectively according to the above regulated evaluation standards. Making lowest score and the highest score value 0 and 1 (or vice versa) is to solve the whitening value of such index. The whitening value are solved according to Fig. 3 and Fig. 4 showed below.

Suppose there are g backup suppliers, qualitative index A, $\mathrm{H}$ experts make scores on it respectively according to the evaluation standards.

If index $\mathrm{A}$ is extremely large index-- the larger the indexes value the better

When $y=y_{\max }$, whitening value $x=1$

When $y=y_{\min }$, whitening value $x=0$

If index A is extremely small index-- the smaller the indexes value the better, then

when $y=y_{\max }$, whitening value $x=0$

when $y=y_{\min }$, whitening value $x=1$

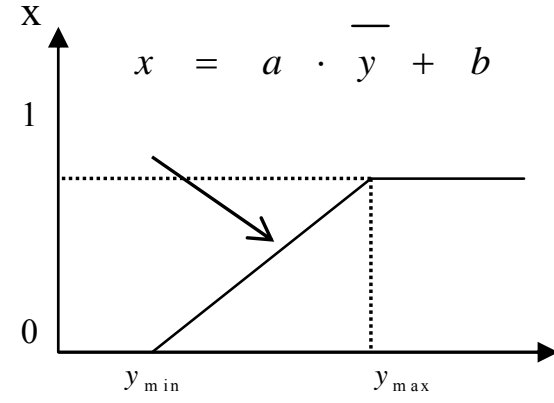

Fig.3 Extremely large type



Fig.4 Extremely large type

The fourth step, calculating grey correlation coefficient and grey correlation degree. 
Correlation data $\beta_{i j}$ is difference value in the number $\mathrm{j}$ index of $\left\{Z_{i}\right\}$ which is comparison sequence of number of backup supplier evaluation index to $\left\{Z_{0}\right\}$ which is the optimal reference data. The difference value can be used as yard stick of correlation degree. The larger the $\beta_{i j}$, the more correlated the two sequence are in the number $\mathrm{j}$ index.

$$
\beta_{i j}=\frac{\min _{i} \min _{j} \Delta_{i j}+\rho \max _{i} \max _{j} \Delta_{i j}}{\Delta_{i j}+\rho \max _{i} \max _{j} \Delta_{i j}} .
$$

In the formula $\Delta_{i j}=\left|z_{0 j}-z_{i j}\right|, \rho$ is called Distinguishing Coefficient. It is for weakening the effects of distortion due to over large of the largest absolute difference. The smaller the value of $\rho$ is, the greater the distinguishing capability. It is chosen between 0 and 1 , usually $\rho=0.5$. the chosen value of $\rho$ does not change correlation degree [2].

Because correlation coefficient can only reflect correlation degree of comparison data column with the optimal reference data in one selection and evaluation index, it can not embody the advantage and disadvantage of backup suppliers. So we can use correlation degree to embody it, correlation degree between comparison data column $\left\{Z_{i}\right\}$ and the optimal reference data $\left\{Z_{0}\right\}$ is

$$
\theta_{i}=1 / n \sum_{i=1}^{n} \omega_{i} \beta_{i j}
$$

Fifth step, calculating grey correlation degree of backup suppliers according to the above steps and getting the results in order. The larger the grey correlation degree value $\theta_{i}$ is the more close comparison data column $\left\{Z_{i}\right\}$ and the optimal reference data $\left\{Z_{0}\right\}$ is in space shape. That is, the more powerful the backup supplier is in overall strength. Since it could be chosen as important partner model for strategic supplier for manufacturing enterprise.

\section{Sample}

Located in Anhui province, C Automobile Science and Technology Corporation Ltd which integrated vehicle designing and manufacturing is comprehensive business corporation established in 2003 and invested by both of one Chinese finished automobile enterprise and one Canadian automobile parts enterprise. It is in the leading place in the field of vehicle power-operated sliding door, and provides its products to one MPV vehicle manufacturer. The corporation applied the above mentioned strategic supplier selection and evaluation system of manufacturing enterprise in the work of selection and evaluation of power-operated sliding door backup supplier and did a nice job.

$\mathrm{C}$ corporation chose staff from department of purchasing, research and development, and production to organize evaluation team for sliding door drive system supplier selection. Then they decided 5 suppliers distributed Yangtze River delta as the backup suppliers after a round of primary election. Drawing lessons from the suppliers relationship management mode of General Motors, C corporation carried out on-site investigation of one week to collect related information and use AHP-GCA (analytic hierarchy process-grey correlation analysis) to carry out selection and evaluation to achieve the advantages and disadvantages of these suppliers.

First, constructing multiple judgment matrix, scoring by experts through nine points ratio method, calculating weighting of various indexes and carrying out consistency check. Table 1 shows that building 7 first level indexes and 23 second level indexes based on selection and evaluation index system. Various indexes and weighting at Index level B and level $\mathrm{C}$ and consistency check results are showed respectively from Table 2 to Table 9. 
Table 1. C level indexes 's combined weighting towards target A

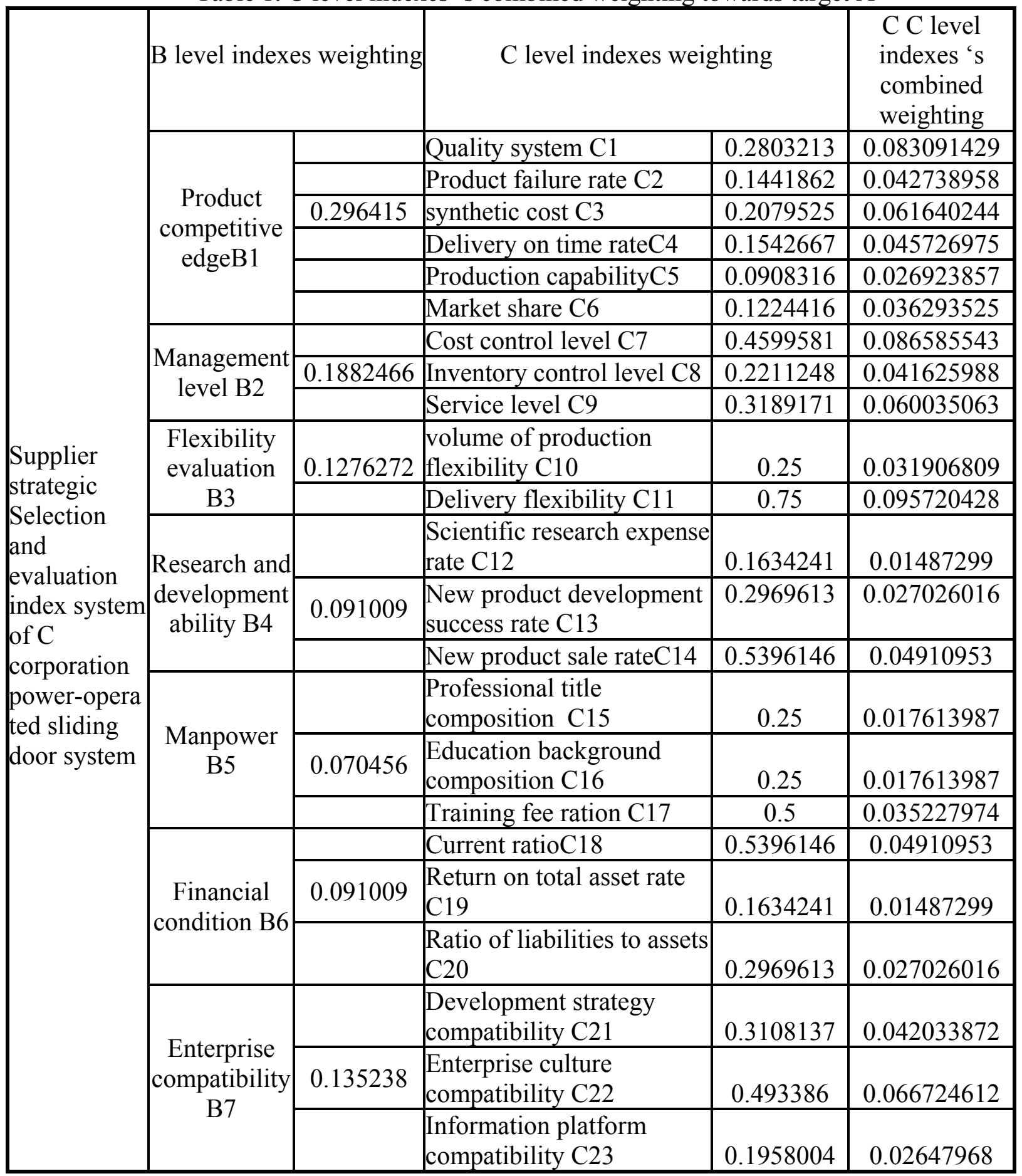

Table 2. Various indexes and weighting at Index level B and consistency check

\begin{tabular}{|c|c|c|c|c|c|c|c|c|c|}
\hline A & B1 & B2 & B3 & B4 & B5 & B6 & B7 & $\omega_{\mathrm{i}}$ & $\lambda_{\mathrm{i}}$ \\
\hline B1 & 1 & 2 & 3 & 3 & 3 & 2 & 3 & 0.296415 & 1.02554815 \\
\hline B2 & $1 / 2$ & 1 & 1 & 1 & 3 & 3 & 3 & 0.1882466 & 1.09673607 \\
\hline B3 & $1 / 3$ & 1 & 1 & 2 & 2 & 2 & $1 / 3$ & 0.1276272 & 1.07982352 \\
\hline B4 & $1 / 3$ & 1 & $1 / 2$ & 1 & 1 & 1 & $1 / 2$ & 0.0910085 & 1.05320939 \\
\hline B5 & $1 / 3$ & $1 / 3$ & $1 / 2$ & 1 & 1 & $1 / 2$ & $1 / 2$ & 0.0704559 & 1.0137141 \\
\hline B6 & $1 / 2$ & $1 / 3$ & $1 / 2$ & 1 & 1 & 1 & 1 & 0.0910085 & 1.03990428 \\
\hline B7 & $1 / 3$ & $1 / 3$ & 3 & 2 & 2 & 1 & 1 & 0.1352382 & 1.15522256 \\
\hline \multicolumn{7}{|c|}{ max $=7.46415806$} & \multicolumn{7}{c|}{ Cl=0.07735968 } & & 1 & \\
\hline
\end{tabular}


Table 3. Various indexes and weighting at Index level $\mathrm{C}$ and consistency check

\begin{tabular}{|c|c|c|c|c|c|c|c|c|}
\hline B1 & $\mathrm{C} 1$ & $\mathrm{C} 2$ & $\mathrm{C} 3$ & $\mathrm{C} 4$ & $\mathrm{C} 5$ & $\mathrm{C6}$ & $\omega i$ & $\lambda \mathrm{i}$ \\
\hline C1 & 1 & 3 & 1 & 2 & 3 & 2 & 0.280321 & 1.038537 \\
\hline $\mathrm{C} 2$ & $1 / 3$ & 1 & 1 & 1 & 2 & 1 & 0.144186 & 1.044888 \\
\hline $\mathrm{C} 3$ & 1 & 1 & 1 & 1 & 3 & 2 & 0.207953 & 0.7872638 \\
\hline $\mathrm{C} 4$ & $1 / 2$ & 1 & 1 & 1 & 1 & 2 & 0.154267 & 1.0612364 \\
\hline $\mathrm{C5}$ & $1 / 3$ & $1 / 2$ & $1 / 2$ & 1 & 1 & $1 / 2$ & 0.090832 & 1.0565867 \\
\hline $\mathrm{C} 6$ & $1 / 2$ & 1 & $1 / 2$ & $1 / 2$ & 2 & 1 & 0.122442 & 1.0475217 \\
\hline \multicolumn{4}{|c|}{$\lambda \max =6.0360337$} & \multicolumn{3}{|c|}{$\mathrm{Cl}=0.0072067$} & 1 & \\
\hline
\end{tabular}

Table 4. Various indexes and weighting at Index level $\mathrm{C}$ and consistency check

\begin{tabular}{|c|c|c|c|c|c|}
\hline B2 & C7 & C8 & C9 & $\omega \mathrm{i}$ & $\lambda \mathrm{i}$ \\
\hline C7 & 1 & 3 & 1 & 0.46 & 1.05 \\
\hline C8 & $1 / 3$ & 1 & 1 & 0.221 & 1.05 \\
\hline C9 & 1 & 1 & 1 & 0.319 & 1.05 \\
\hline$\lambda \max =3.14$ & \multicolumn{2}{|c|}{$\mathrm{Cl}=0.07$} & 1 & \\
\hline
\end{tabular}

Table 6.Various indexes and weighting at Index level $\mathrm{C}$ and consistency check

\begin{tabular}{|c|c|c|c|c|c|}
\hline $\mathrm{B} 4$ & $\mathrm{C} 12$ & $\mathrm{C} 13$ & $\mathrm{C} 14$ & $\omega \mathrm{i}$ & $\mathrm{\lambda} \mathrm{i}$ \\
\hline $\mathrm{C} 12$ & 1 & $1 / 2$ & $1 / 3$ & 0.163 & 1 \\
\hline $\mathrm{C} 13$ & 2 & 1 & $1 / 2$ & 0.297 & 1 \\
\hline $\mathrm{C} 14$ & 3 & 2 & 1 & 0.54 & 1 \\
\hline$\lambda \max =3.01$ & \multicolumn{3}{|c|}{$\mathrm{Cl}=0$} & 1 & \\
\hline
\end{tabular}

Table 8. Various indexes and weighting at Index level $\mathrm{C}$ and consistency check

\begin{tabular}{|c|c|c|c|c|c|}
\hline B6 & $\mathrm{C} 18$ & $\mathrm{C} 19$ & $\mathrm{C} 20$ & $\omega \mathrm{i}$ & $\lambda \mathrm{i}$ \\
\hline $\mathrm{C} 18$ & 1 & 3 & 2 & 0.54 & 1.0031 \\
\hline $\mathrm{C} 19$ & $1 / 3$ & 1 & $1 / 2$ & 0.163 & 1.0031 \\
\hline $\mathrm{C} 20$ & $1 / 2$ & 2 & 1 & 0.297 & 1.0031 \\
\hline$\lambda \max =3.0092$ & $\mathrm{Cl}=0.0046$ & 1 & \\
\hline
\end{tabular}

Table 5.various indexes and weighting at Index level $\mathrm{C}$ and consistency check

\begin{tabular}{|c|c|c|c|c|}
\hline B3 & $\mathrm{C} 10$ & $\mathrm{C} 11$ & $\omega \mathrm{i}$ & $\lambda \mathrm{i}$ \\
\hline $\mathrm{C} 10$ & 1 & $1 / 3$ & 0.25 & 1 \\
\hline $\mathrm{C} 11$ & 3 & 1 & 0.75 & 1 \\
\hline & & & 1 & \\
\hline \multicolumn{2}{|c|}{$\lambda \max =2$} & $\mathrm{Cl}=0$ & & \\
\hline
\end{tabular}

Table 7.Various indexes and weighting at Index level $\mathrm{C}$ and consistency check

\begin{tabular}{|c|c|c|c|c|c|}
\hline $\mathrm{B} 5$ & $\mathrm{C} 15$ & $\mathrm{C} 16$ & $\mathrm{C} 17$ & $\omega \mathrm{i}$ & $\lambda \mathrm{i}$ \\
\hline $\mathrm{C} 15$ & 1 & 1 & $1 / 2$ & 0.25 & 1 \\
\hline $\mathrm{C} 16$ & 1 & 1 & $1 / 2$ & 0.25 & 1 \\
\hline $\mathrm{C} 17$ & 2 & 2 & 1 & 0.5 & 1 \\
\hline$\lambda \max =3.01$ & \multicolumn{2}{|c|}{$\mathrm{Cl}=0$} & 1 & \\
\hline
\end{tabular}

Table 9.Various indexes and weighting at Index level $\mathrm{C}$ and consistency check

\begin{tabular}{|c|c|c|c|c|c|}
\hline $\mathrm{B} 7$ & $\mathrm{C} 21$ & $\mathrm{C} 22$ & $\mathrm{C} 23$ & $\omega \mathrm{i}$ & $\lambda \mathrm{i}$ \\
\hline $\mathrm{C} 21$ & 1 & $1 / 2$ & 2 & 0.311 & 1.0179 \\
\hline $\mathrm{C} 22$ & 2 & 1 & 2 & 0.493 & 1.0179 \\
\hline $\mathrm{C} 23$ & $1 / 2$ & $1 / 2$ & 1 & 0.196 & 1.0179 \\
\hline \multicolumn{2}{|l}{$\max =3.0536$} & $\mathrm{Cl}=0.0268$ & 1 & \\
\hline
\end{tabular}

Second, carrying out standardization and whitening weight function on five backup suppliers, see table 10. 
Table 10. Power-operated sliding door suppliers original data/comparison sequence of number

\begin{tabular}{|c|c|c|c|c|c|c|c|c|c|c|c|}
\hline Suppliers & \multicolumn{2}{|l|}{$\bar{A}$} & \multicolumn{2}{|l|}{$\mathrm{B}$} & \multicolumn{2}{|l|}{$\mathrm{C}$} & \multicolumn{2}{|l|}{$\bar{D}$} & \multicolumn{2}{|l|}{$E$} & \multirow[b]{2}{*}{$\mathrm{Z}_{0}$} \\
\hline $\begin{array}{c}\text { original } \\
\text { data/comparison } \\
\text { sequence of number }\end{array}$ & Original & $Z_{1}$ & Original & $\mathrm{Z}_{2}$ & Original & $\mathrm{Z}_{3}$ & Original & $\mathrm{Z}_{4}$ & Original & $\mathrm{Z}_{5}$ & \\
\hline Quality system C1 & 9 & 1 & 8 & 0.5 & 9 & 1 & 9 & 1 & 8 & 0.5 & 1 \\
\hline Product $b$ defect rate $\mathrm{C} 2$ & 300 & 1 & 350 & 0.88 & 400 & 0.75 & 300 & 1 & 350 & 0.88 & 1 \\
\hline Synthetic cost $\mathrm{C} 3$ & 3780 & 0.98 & 3880 & 0.95 & 3960 & 0.93 & 3690 & 1 & 3750 & 0.99 & 1 \\
\hline $\begin{array}{l}\text { Delivery on time rate } \\
\text { C4 }\end{array}$ & $98 \%$ & 0.99 & $99 \%$ & 1 & $99 \%$ & 1 & $97 \%$ & 0.98 & $98 \%$ & 0.99 & 1 \\
\hline Production capacity C5 & 100 & 0.5 & 200 & 1 & 150 & 0.75 & 130 & 0.65 & 160 & 0.8 & 1 \\
\hline Market share C6 & $15 \%$ & 0.83 & $18 \%$ & 1 & $10 \%$ & 0.56 & $8 \%$ & 0.44 & $12 \%$ & 0.67 & 1 \\
\hline Cost control level C7 & $130 \%$ & 0.96 & $125 \%$ & 0.93 & $135 \%$ & 1 & $128 \%$ & 0.95 & $120 \%$ & 0.89 & 1 \\
\hline $\begin{array}{c}\text { Inventory control level } \\
\text { C8 } \\
\end{array}$ & $86 \%$ & 0.59 & $108 \%$ & 0.74 & $133 \%$ & 0.91 & $120 \%$ & 0.82 & $147 \%$ & 1 & 1 \\
\hline Service level C9 & $13 \%$ & 0.62 & $10 \%$ & 0.85 & $8 \%$ & 1 & $9 \%$ & 0.92 & $11 \%$ & 0.77 & 1 \\
\hline $\begin{array}{c}\text { Volume of production } \\
\text { flexibility } \mathrm{C} 10\end{array}$ & $80 \%$ & 0.88 & $87 \%$ & 0.96 & $91 \%$ & 1 & $86 \%$ & 0.95 & $78 \%$ & 0.86 & 1 \\
\hline Delivery flexibility C11 & $28 \%$ & 0.93 & $30 \%$ & 1 & $22 \%$ & 0.73 & $25 \%$ & 0.83 & $23 \%$ & 0.77 & 1 \\
\hline $\begin{array}{l}\text { Scientific research } \\
\text { expense rate } \mathrm{C} 12\end{array}$ & $5 \%$ & 1 & $3 \%$ & 0.6 & $4 \%$ & 0.8 & $2 \%$ & 0.4 & $5 \%$ & 1 & 1 \\
\hline $\begin{array}{c}\text { New product } \\
\text { development success } \\
\text { rate } \mathrm{C} 13\end{array}$ & $70 \%$ & 0.92 & $68 \%$ & 0.9 & $76 \%$ & 1 & $59 \%$ & 0.78 & $63 \%$ & 0.83 & 1 \\
\hline $\begin{array}{c}\text { New product sale ratio } \\
\mathrm{C} 14\end{array}$ & $10 \%$ & 0.67 & $12 \%$ & 0.8 & $8 \%$ & 0.53 & $15 \%$ & 1 & $11 \%$ & 0.73 & 1 \\
\hline $\begin{array}{l}\text { Professional title } \\
\text { composition C15 }\end{array}$ & $38 \%$ & 0.88 & $42 \%$ & 0.98 & $40 \%$ & 0.93 & $39 \%$ & 0.91 & $43 \%$ & 1 & 1 \\
\hline $\begin{array}{c}\text { Education background } \\
\text { composition } \mathrm{C} 16\end{array}$ & $68 \%$ & 0.93 & $71 \%$ & 0.97 & $66 \%$ & 0.9 & $68 \%$ & 0.93 & $73 \%$ & 1 & 1 \\
\hline Training fee ratio $\mathrm{C} 17$ & $0.20 \%$ & 0.67 & $0.30 \%$ & 1 & $0.25 \%$ & 0.83 & $0.23 \%$ & 0.77 & $0.19 \%$ & 0.63 & 1 \\
\hline Liquidity ratio $\mathrm{C} 18$ & 2 & 0.89 & 2.1 & 0.93 & 1.95 & 0.87 & 2.2 & 0.98 & 2.25 & 1 & 1 \\
\hline $\begin{array}{c}\text { Return on total asset } \\
\text { rate } \mathrm{C} 19\end{array}$ & $12 \%$ & 1 & $11 \%$ & 0.92 & $10 \%$ & 0.83 & $11 \%$ & 0.92 & $12 \%$ & 1 & 1 \\
\hline $\begin{array}{c}\text { Ratio of liabilities to } \\
\text { assets C } 20\end{array}$ & $45 \%$ & 0.88 & $48 \%$ & 0.81 & $39 \%$ & 1 & $42 \%$ & 0.94 & $43 \%$ & 0.92 & 1 \\
\hline \begin{tabular}{|c|} 
Development strategy \\
compatibility C 21
\end{tabular} & 7 & 0.5 & 8 & 1 & 7 & 0.5 & 8 & 1 & 7 & 0.5 & 1 \\
\hline $\begin{array}{l}\text { Enterprise culture } \\
\text { compatibility C22 }\end{array}$ & 8 & 1 & 7 & 0.5 & 7 & 0.5 & 8 & 1 & 7.5 & 0 & 1 \\
\hline $\begin{array}{c}\text { Information platform } \\
\text { compatibility } \mathrm{C} 23\end{array}$ & 8 & 0.5 & 9 & 1 & 8 & 0.5 & 8 & 0.5 & 9 & 1 & 1 \\
\hline
\end{tabular}


Third, solving grey correlation coefficient $\beta_{i j}$ and grey correlation degree $\theta_{i}$, see Table 11 .

Table 11. Power-operated sliding door suppliers $\Delta_{i j}, \beta_{i j}$ and $\theta_{i}(\rho=0.5)$

\begin{tabular}{|c|c|c|c|c|c|c|c|c|c|c|}
\hline suppliers & \multicolumn{2}{|c|}{ A } & \multicolumn{2}{|c|}{$\mathrm{B}$} & \multicolumn{2}{|c|}{$\mathrm{C}$} & \multicolumn{2}{|c|}{$\mathrm{D}$} & \multicolumn{2}{|c|}{$\mathrm{E}$} \\
\hline $\begin{array}{c}\text { comparison } \\
\text { sequence of number }\end{array}$ & $\beta \mathrm{i} 1$ & $\Delta \mathrm{i}(1)$ & $\beta \mathrm{i} 2$ & $\Delta \mathrm{i}(2)$ & $\beta \mathrm{i} 3$ & $\Delta \mathrm{i}(3)$ & $\beta \mathrm{i} 4$ & $\Delta \mathrm{i}(4)$ & $\beta \mathrm{i} 5$ & $\Delta \mathrm{i}(5)$ \\
\hline $\begin{array}{l}\text { Quality system } \\
\text { C1 }\end{array}$ & 1.000 & 0 & 0.357 & 0.5 & 1.000 & 0 & 1.000 & 0 & 0.357 & 0.5 \\
\hline $\begin{array}{l}\text { Product } b \text { defect } \\
\text { rate } \mathrm{C} 2\end{array}$ & 1.000 & 0 & 0.690 & 0.13 & 0.527 & 0.25 & 1.000 & 0 & 0.690 & 0.13 \\
\hline $\begin{array}{c}\text { Synthetic cost } \\
\text { C3 }\end{array}$ & 0.924 & 0.02 & 0.853 & 0.05 & 0.803 & 0.07 & 1.000 & 0 & 0.949 & 0.02 \\
\hline $\begin{array}{c}\text { Delivery on time } \\
\text { rate } \mathrm{C} 4\end{array}$ & 0.965 & 0.01 & 1.000 & 0 & 1.000 & 0 & 0.933 & 0.02 & 0.965 & 0.01 \\
\hline \begin{tabular}{|} 
Production capacity \\
C5 \\
\end{tabular} & 0.357 & 0.5 & 1.000 & 0 & 0.527 & 0.25 & 0.443 & 0.35 & 0.582 & 0.2 \\
\hline $\begin{array}{c}\text { Market share } \\
\text { C6 } \\
\end{array}$ & 0.625 & 0.17 & 1.000 & 0 & 0.385 & 0.44 & 0.333 & 0.556 & 0.455 & 0.33 \\
\hline \begin{tabular}{|c|} 
Cost control level \\
C7 \\
\end{tabular} & 0.883 & 0.04 & 0.790 & 0.07 & 1.000 & 0 & 0.842 & 0.05 & 0.715 & 0.11 \\
\hline \begin{tabular}{|c|} 
Inventory control \\
level C8 \\
\end{tabular} & 0.401 & 0.42 & 0.512 & 0.27 & 0.745 & 0.1 & 0.602 & 0.18 & 1.000 & 0 \\
\hline $\begin{array}{c}\text { Service level } \\
\text { C9 } \\
\end{array}$ & 0.419 & 0.39 & 0.644 & 0.15 & 1.000 & 0 & 0.783 & 0.08 & 0.546 & 0.23 \\
\hline $\begin{array}{c}\text { Volume of } \\
\text { production } \\
\text { flexibility C10 }\end{array}$ & 0.697 & 0.12 & 0.863 & 0.04 & 1.000 & 0 & 0.835 & 0.06 & 0.660 & 0.14 \\
\hline $\begin{array}{c}\text { Delivery flexibility } \\
\text { C11 } \\
\end{array}$ & 0.806 & 0.07 & 1.000 & 0 & 0.510 & 0.27 & 0.625 & 0.17 & 0.544 & 0.23 \\
\hline $\begin{array}{l}\text { Scientific research } \\
\text { expense rate } \mathrm{C} 12\end{array}$ & 1.000 & 0 & 0.410 & 0.4 & 0.582 & 0.2 & 0.357 & 0.6 & 1.000 & 0 \\
\hline $\begin{array}{c}\text { New product } \\
\text { development } \\
\text { success rate } \mathrm{C} 13\end{array}$ & 0.779 & 0.08 & 0.726 & 0.11 & 1.000 & 0 & 0.554 & 0.22 & 0.619 & 0.17 \\
\hline \begin{tabular}{|c|} 
New product sale \\
ratio $\mathrm{C} 14$
\end{tabular} & 0.455 & 0.33 & 0.582 & 0.2 & 0.373 & 0.47 & 1.000 & 0 & 0.510 & 0.27 \\
\hline $\begin{array}{l}\text { Professional title } \\
\text { composition C15 }\end{array}$ & 0.706 & 0.12 & 0.924 & 0.02 & 0.799 & 0.07 & 0.749 & 0.09 & 1.000 & 0 \\
\hline $\begin{array}{c}\text { Education } \\
\text { background } \\
\text { composition } \mathrm{C} 16\end{array}$ & 0.803 & 0.07 & 0.911 & 0.03 & 0.743 & 0.1 & 0.803 & 0.07 & 1.000 & 0 \\
\hline $\begin{array}{c}\text { Training fee ratio } \\
\text { C17 } \\
\end{array}$ & 0.455 & 0.33 & 1.000 & 0 & 0.625 & 0.17 & 0.544 & 0.23 & 0.431 & 0.37 \\
\hline $\begin{array}{c}\text { Liquidity ratio } \\
\text { C18 }\end{array}$ & 0.715 & 0.11 & 0.806 & 0.07 & 0.676 & 0.13 & 0.927 & 0.02 & 1.000 & 0 \\
\hline $\begin{array}{l}\text { Return on total } \\
\text { asset rate C19 }\end{array}$ & 1.000 & 0 & 0.770 & 0.08 & 0.625 & 0.17 & 0.770 & 0.08 & 1.000 & 0 \\
\hline $\begin{array}{c}\text { Ratio of liabilities } \\
\text { to assets C20 } \\
\end{array}$ & 0.690 & 0.13 & 0.598 & 0.19 & 1.000 & 0 & 0.818 & 0.06 & 0.770 & 0.08 \\
\hline
\end{tabular}




\begin{tabular}{|c|c|c|c|c|c|c|c|c|c|c|}
\hline $\begin{array}{c}\text { Development } \\
\text { strategy } \\
\text { compatibility C21 }\end{array}$ & 0.357 & 0.5 & 1.000 & 0 & 0.357 & 0.5 & 1.000 & 0 & 0.357 & 0.5 \\
\hline $\begin{array}{c}\text { Enterprise culture } \\
\text { compatibility C22 }\end{array}$ & 1.000 & 0 & 0.357 & 0.5 & 0.357 & 0.5 & 1.000 & 0 & 0.357 & 0.5 \\
\hline $\begin{array}{c}\text { Information } \\
\text { platform } \\
\text { compatibility C23 }\end{array}$ & 0.357 & 0.5 & 1.000 & 0 & 0.357 & 0.5 & 0.357 & 0.5 & 1.000 & 0 \\
\hline min $\triangle \mathrm{ij}$ & & 0 & & 0 & & & & 0 & & 0 \\
\hline $\max \triangle \mathrm{ij}$ & & 0.5 & & 0.5 & & 0.5 & & 0.556 & & 0.5 \\
\hline$\theta \mathrm{i}$ & 0.032 & & 0.033 & & 0.031 & & 0.035 & & 0.029 & \\
\hline
\end{tabular}

Fourth, sequence according to grey correlation degree.

According to grey correlation degree $\theta_{i}$ from large value to small value, $\theta_{4}>\theta_{2}>\theta_{1}>\theta_{3}>\theta_{5}$.

Among five backup power-operated sliding door drive system suppliers of $\mathrm{C}$ corporation, $\mathrm{D}$ supplier is benchmarking. Of course, D supplier with the largest correlation degree may not be the one which suits $\mathrm{C}$ corporation best. $\mathrm{C}$ corporation has to choose alternatives, $\mathrm{B}$ and $\mathrm{A}$ can be chosen as backup alternatives. In the actual work, $\mathrm{C}$ corporation finally chooses D supplier as its strategic supplier of power-operated sliding door.

\section{Conclusion}

Strategic supplier selection is one of the important questions within supply chain in manufacturing industry. The integrated AHP-GCA method for supplier selection and evaluation is effective. First, using the method of AHP to calculate the weights of the first level indexes and the second level indexes and checking the consistency in order to combine the experience of industry experts with their rational thinking. Second, through standardization and whitening weight function of quantitative and qualitative data, the comparison data of optional suppliers are formed. Then using grey correlation method to solve correlation coefficient of various optional suppliers by combining the weights of all indexes. In this way, the order of advantage and disadvantage are confirmed. It makes great achievement in quantitative and qualitative decisions and grey information disposal in actual application of selection and evaluation of optional strategic supplier in manufacturing industries.

\section{References}

[1] Hu Yunquan, Operations Research, Tsinghua University Press, Beingjing, 2003.

[2] Deng Julong, Grey Prediction and Decision, Huazhong University of Science and Technology press, Wuhan, 1986.

[3] Liu Sifeng, CaiHua, Yang Yingjie, Cao Ying, Advanced in Grey Incidence Analysis Modelin, J. System Engineering Theory\& Practice. 8(2013) 2041-2046. 